3 Randic M, Miletic V. Depressant actions of methionine-enkephalin and somatostatin in cat dorsa horn neurones activated by noxious stimuli. Brain Res 1978;152:196-202.

4 Hökfelt T, Kellerth JO, Nilsson G, Pernow B. Experimental immunohistochemical studies on the localization and distribution of substance $\mathrm{P}$ in cat primary sensory neurones. Brain Res 1975; 100:235-52.

5 Takagi H, Shiomi H, Fukui K, Hayashi K, Kiso Y, Kitagawa K. Isolation of a novel analgesic pentapeptide, neokyotorphin, from bovine brain. Life Sci 1982;31:1733-6.

(Accepted 11 June 1987)

Department of Medicine, Royal Postgraduate Medical School, London W12 0HS

GARETH WILLIAMS, MD, MRCP, R D Lawrence research fellow, honorary senior registrar

JOANNA A BALL, MB, MRCP, research registrar

RODERICK A LAWSON, MB, senior house officer

GRAHAM F JOPLIN, PHD, FRCP, professor of clinical endocrinology

STEPHEN R BLOOM, MD, FRCP, professor of endocrinology

St Bernard's Hospital, Gibraltar

MICHAEL R MASKILL, MB, MRCP, consultant physician

Correspondence to: Professor Bloom.

\section{Reactive arthritis associated with cryptosporidium enteritis}

Enteric infection with cryptosporidium is now increasingly recognised as a cause of diarrhoea in man. The incidence appears to vary geographically and throughout the year, cases being reported most commonly in the late summer and early autumn. ${ }^{1}$ In immunocompetent patients the illness is self limiting, lasting from seven to 10 days, but immunocompromised patients may be severely affected with life threatening and protracted diarrhoea. ${ }^{2}$ There is no effective antimicrobial treatment. The clinical manifestation of watery, non-bloody diarrhoea is not specific to this organism. Vomiting is common initially together with abdominal pain, which is sometimes severe and may precede the diarrhoea. Although a malabsorption syndrome may occasionally follow the acute episode, there have been no other long term sequelae in immunocompetent patients. We describe a case of cryptosporidium enteritis associated with reactive arthritis.

\section{Case report}

A 27 year old man was admitted from the casualty department, where he had presented with an acutely swollen, painful right knee of 24 hours' duration and arthralgias affecting his neck, lower back, and left knee for 12 hours. He had no previous history of joint symptoms. He had been well until seven days before admission, when he had developed colicky, abdominal pain associated with anorexia, nausea, and vomiting followed by two days of severe, watery diarrhoea for which he had been prescribed loperamide. A neighbour (aged 5 years) had also had diarrhoea one week. earlier. There was no history of conjunctivitis or urethritis.

On examination the right knee was hot, swollen, tender, held in slight flexion, and extremely painful when moved. After admission he had no further bowel symptoms but continued to have severe arthralgias, which progressed to include his wrists and left heel. There was a persistent effusion of the right knee. Treatment was symptomatic with naproxen in conventional dosage and bed rest, but it was only partly effective. He was discharged after one week, but on review a week later he had persistent arthralgias and increasing pain in his right knee. The joint was aspirated and, after a culture of the synovial fluid gave negative results, it was injected with steroid and it subsequently settled. He improved slowly, but four months later he still had mild arthralgia, particularly in his knees.

The results of the following investigations were normal or negative: full blood count; electrolytes; immunoglobulins; rheumatoid factor; autoantibodies; antistreptolysin $\mathrm{O}$ titre; and antibodies to Campylobacter jejuni, Yersinia enterocolitica Salmonella typhi, and $S$ paratyphi. The erythrocyte sedimentation rate was $66 \mathrm{~mm}$ in the first hour. Examination of a rectal swab showed no evidence of shigella, campylobacter, or salmonella, but oocysts of cryptosporidium were identified Human leucocyte antigen B27 (HLA-B27) typing was negative.

\section{Comment}

In this patient the temporal relation between the diarrhoea and the arthropathy, together with the sterile effusion, suggested reactive arthritis. Although this is well recognised after infection with a number of other intestinal pathogens, including salmonella, shigella, $Y$ enterocolitica, and $C$ jejuni, ${ }^{34}$ there are no reports of reactive arthritis secondary to infection with cryptosporidium. Only a few patients with bowel infection develop reactive arthritis. Genetic factors, including the presence of HLA-B27, are thought to play a part in the pathogenesis and clinical expression of the spond- arthritides. HLA-B27 is present in up to $63 \%$ of patients with Reiter's syndrome and it correlates with uveitis, severe constitutional upset, and a chronic or relapsing course. ${ }^{5}$ The underlying mechanisms are not completely understood, and the occurrence of reactive arthritis in a patient negative for HLA-B27 suggests that an additional gene or genes may be implicated. Since techniques have become available for the rapid detection of cryptosporidium oocysts in the stools an increasing number of laboratories are including these tests in the routine investigation of patients with diarrhoea. Following this report cryptosporidium infection should be considered as a possible causal agent in patients with diarrhoea that gives negative results on culture and reactive arthritis.

1 Bissenden JG. Cryptosporidium and diarrhoea. Br Med J 1986;293:287-8.

2 Malebranche R, Arnovx E, Guerin JM, et al. Acquired immunodeficiency syndrome with severe gastrointestinal manifestation. Lancet 1983;ii:487-9.

3 Larsen JH. Yersinia enterocolitica infections and arthritis. In: Dumonde DC, ed. Infection and immunology in the rheumatic diseases. Oxford: Blackwell, 1976;17:133-40.

4 Kosunen TU, Pönkä A, Kauraneno O, el al. Arthritis associated with campylobacter enteritis. Scand I Rheumatol 1981;10:77-80.
Sosunen TU, Ponka A, Kauraneno

5 McClusky OE, Lordon RE, Arnett FC. HL-A 27 in Reiter's syndrome and psoriatic arthritis: a genetic factor in disease susceptibility and expression. $\mathcal{F}$ R heumatol 1984;11:571.

(Accepted 24 April 1987)

Department of Rheumatology, Nether Edge Hospital, Sheffield S11 9EL

E M HAY, MRCP, registrar in rheumatology

J WINFIELD, MRCP, consultant in rheumatology and rehabilitation

Department of Medicine and Communicable Diseases, Lodge Moor Hospital, Sheffield

M W McKENDRICK, MRCP, consultant physician

Correspondence to: Dr Winfield.

\section{A simple and effective treatment for AIDS related Kaposi's sarcoma}

About a third of British patients with the acquired immune deficiency syndrome (AIDS) have Kaposi's sarcoma, ${ }^{1}$ which is usually diagnosed before there is evidence of immune deficiency. Characteristically the lesions are purplish blue infiltrated patches that may occur on all parts of the skin and mucous membranes, especially the trunk, face, penis, and palate. Though the disease may pursue an aggressive course, it is usually only a disfiguring and distressing precursor of and accompaniment to the opportunistic infections of which patients with AIDS usually die.

Epidemic Kaposi's sarcoma may be treated with chemotherapy, immunotherapy, or radiotherapy. ${ }^{2 \cdot 4}$ Local radiotherapy has minimal side effects and is an effective way of producing regression of individual lesions. Furthermore, it does not cause immunosuppression and so does not affect the prognosis of either the AIDS or the generalised Kaposi's sarcoma. Reduction in size and control of enlarging lesions, especially on the face or feet, may appreciably improve the quality of life of patients with AIDS. We describe the first series reported from Britain of patients with epidemic Kaposi's sarcoma treated with local radiotherapy by means of a single fraction irradiation technique.

Patients, methods, and results

Patients with Kaposi's sarcoma were referred for possible radiotherapy by their general practitioners, the department of medicine and genitourinary medicine at this hospital, and the St John's Hospital for Diseases of the Skin, London. The indications for irradiation were an increase in size of the tumours, lesions that were bleeding, particularly on the feet (which may not only restrict mobility but are also a potential source of infection), and pedunculated and ulcerated palatal lesions. These last cause soreness of the mouth, instability of teeth, and eventually dyspnoea and dysphagia. Small cosmetically distressing lesions on the face were also treated.

The table gives details of the patients treated, dose of radiation and number of treatment sessions, and outcome. In all patients radiotherapy resulted in at least partial regression of all the lesions treated.

\section{Comment}

The lesions of Kaposi's sarcoma in patients with AIDS are seldom life threatening but frequently cause considerable discomfort and emotional distress. Local palliative irradiation of troublesome patches is an effective way of controlling these lesions without causing immunosuppression.

Our experience suggests the following approach to treatment. Lesions 